\title{
ASCLS Annual Meeting 2011: Official Abstracts of Submitted Papers, Case Studies and Posters
}

\begin{abstract}
The following abstracts have been accepted for presentation at the 2011 American Society for Clinical Laboratory Science (ASCLS) Annual Meeting and Clinical Laboratory Exposition to be held July 26 through July 30 in Atlanta, GA. Abstracts are reviewed by appropriate representatives of the ASCLS Abstract Review Committee. They are the final authority in selecting or rejecting an abstract.
\end{abstract}

Atlanta, GA

Papers, case studies and posters will be presented during the following times at the annual meeting.

\section{ORAL RESEARCH PRESENTATIONS}

Wednesday, July 27, 3:30-4:30pm at the Georgia World Congress Center

Friday, July 29, 9:00-10:00am at the Omni Hotel

\section{POSTER PRESENTATIONS}

Tuesday and Wednesday, July 26 and 27, 10:00am4:30pm; Thursday, July 28, 9:30am-Noon at the Georgia World Congress Center; Authors will be present on Wednesday, July 27, 2011 from 10:30am to Noon to discuss their work and answer questions.

\section{Oral Research Abstracts}

Survey of Healthcare Providers' Expectations of Qualifications of Medical Laboratory Practitioners Michelle Butina, PhD, MLS(ASCP) ${ }^{\mathrm{CM}}$, University of Kentucky, Lexington, KY

The purpose of this study was to survey healthcare providers who order laboratory testing (physicians, physician assistants, and nurse practitioners) to assess their knowledge of the minimum qualifications of medical laboratory practitioners to determine if they are aware of the formal training required to perform laboratory testing. Due to the current laboratory personnel shortage some laboratories have resorted to hiring individuals not qualified and/or formally trained thus significantly increasing the risk of laboratory errors. Since healthcare providers and their patients are greatly impacted by the services of the medical laboratory they should be aware of the quality of this service. A total of 161 brief surveys were delivered to a convenience sample of healthcare providers in Georgia. Eighty-seven healthcare providers (response rate of 54\%) completed these surveys and based on the results, several findings became evident. First, healthcare providers believe that a bachelor's degree should be the primary degree required for those performing laboratory testing. Second, healthcare providers believe practitioners should be certified by a national certifying agency recognizing competence. Last, these providers believe practitioners should have an individual professional license to practice in this state. In summary, this survey of healthcare providers suggest that they believe laboratory testing should be performed by those who have obtained a bachelor's degree and are certified and licensed, yet this is often not the case.

Building an Active and Reflective Laboratory Experience for Clinical Chemistry Students

Janelle M. Chiasera, PhD, Brianna Miller, MS, The University of Alabama at Birmingham, Birmingham, AL

Rapid advances in technology and automation have transformed the practice of clinical chemistry. With fully automated robotic systems, interconnected instrumentation, and on-board applications such as autoverification, the role of the clinical chemistry technologist has evolved to one focused on critical thinking and trouble-shooting. While such advances have transformed the clinical practice of chemistry, the education of students in clinical laboratory science 


\section{CLINICAL PRACTICE}

programs has not progressed as quickly. Consequently, there appears to be a disconnect between student laboratories and the real world practice of chemistry. To attempt to bridge the gap, we have developed a clinical chemistry laboratory teaching method that provides students an opportunity to apply critical thinking and troubleshooting skills to weekly chemistry laboratories. Students are divided into groups, presented with a patient history, and perform chemistry analyses on samples related to their assigned patient on a weekly basis. At completion of the course, each group of students has a complete laboratory report for their patient containing both concordant and discordant data. Twice during the semester, students are required to develop and deliver oral presentations describing their case history, the data collected, a hypothesized and final diagnosis, and are expected to explain concordant and discordant data. This method of instruction promotes critical thinking and troubleshooting skills, engages students more fully into the laboratory experience, and builds a strong base of interpersonal skills.

\section{Correlation of Serum Lactate Levels and Mortality in} Septic Patients

Anne B. Ranne, MS, MT(ASCP), Sead Krndzija, MS, MLS(ASCP), Georgia Health Sciences University, Augusta, GA

The purpose of this study was to demonstrate the statistical significance of serum lactate measurements on mortality rates of patients admitted to an Intensive Care Unit. There is some controversy over using serum lactate measurements as a diagnostic tool. Treatment of severe septic patients within the first few hours following admission is critical in restoring organ function and decreasing mortality. A rapid biomarker test could reduce sepsis deaths from 215,000 per year. This retrospective, cross-sectional study consisted of 30 septic patients admitted to a local hospital's ICU who died within 30 days (non-survivors group) and 30 septic patients who survived (survivors group). Data collection included demographics, serum lactate levels, and infection sources. Only one initial serum lactate was collected, so correlation of serial measurements was not available for this study. The serum lactate range from previous studies was defined as $l o w=<2 \mathrm{mmol} / \mathrm{L}$, intermediate $=2-3.9 \mathrm{mmol} / \mathrm{L}$, and high $=\geq 4 \mathrm{mmol} / \mathrm{L}$. Of the survivors group the initial serum lactate levels were 0.6-6.1 $\mathrm{mmol} / \mathrm{L}$ (mean $2.3 \mathrm{mmol} / \mathrm{L}$ ) and of the nonsurvivors group the lactate levels were $1.6-11.5 \mathrm{mmol} / \mathrm{L}$ (mean $4.8 \mathrm{mmol} / \mathrm{L}$ ). The t-test of the two groups supported a statistical significance between high lactate levels and death of septic patients. The development of a sepsis care bundle including the serum lactate has been suggested by the Surviving Sepsis Campaign. The laboratory staff can be an integral part in reducing sepsis-related mortality by participating in a sepsis response team.

Effectiveness of Educating Nurses as a Means to Decrease the Number of Contaminated Specimens Kyle Riding, CLS, Jennifer Cague, MT(ASCP), Maureen Samson MLS(ASCP) ${ }^{\mathrm{CM}}$, Mark Kellogg, PhD, Children's Hospital Boston, Boston, MA

The purpose of this study was to determine if providing education to nursing staff regarding intravenous fluid contamination could decrease the number of specimens rejected due to contamination. Collecting specimens that are contaminated with IV fluid increases the risk of inaccurate results being released, increases overall turnaround time by requiring specimen recollection, and affects the cost of patient care by requiring extra staff time and equipment. Using LIS software, the cancellation database was queried for a monthly count of specimens from this ward cancelled due to contamination. Per laboratory policy a specimen may be reported to the LIS as contaminated only after a laboratory scientist suspects the presence of IV fluid and confirms this with the patient's nurse. Six months of data was collected via this method and confirmed that this ward had a high rate of specimen contamination. Patient case studies were then prepared that highlighted the effects that different intravenous fluids can have on patient results. These case studies were then presented to senior nurses within the unit. After the presentation of the case studies, the absolute number of specimens rejected due to contamination was again monitored for six months via the same method described above. A statistically significant $(\mathrm{p}<0.05)$ decrease in the absolute number of specimens rejected due to contamination was found to have occurred after the intervention. The researchers concluded that laboratory professionals 


\section{CLINICAL PRACTICE}

could effectively decrease the number of contaminated specimens by working collaboratively with nursing staff and educating them on the importance of specimen integrity.

\section{Two Cases of Anti-M: A Study in Contrasts}

Linda A. Smith, PhD, MLS(ASCP) ${ }^{\mathrm{CM}}$, University of Texas Health Science Center, San Antonio, TX; Tiffany Roerich, MLS(ASCP) ${ }^{\mathrm{CM}}$, CHRISTUS Santa Rosa Hospital, San Antonio, TX

Anti-M is generally considered a clinically significant antibody that can be ignored in routine transfusion practice. However, there are cases in which an $\mathrm{IgG}$ component is present requiring antigen negative blood. This presentation will discuss two cases of three year-old children who were admitted for open heart surgery with orders for type and crossmatch. In both cases anti-M was identified. In the first case where the child had been previously transfused, the anti-M demonstrated a typical cold-reacting pattern. In addition the anti-M caused a backtype discrepancy that had to be resolved. Crossmatching was performed only at AHG in order to obtain compatible units. In the second case in which the patient did not have a history of transfusion, an anti-M that reacted at $37^{\circ} \mathrm{C}$ and AHG was identified. Further testing also demonstrated reactions at immediate spin. Antigen negative units were required for the crossmatch. Child one was transfused with no adverse reaction. Child two did not require blood.

\section{Poster Presentation Abstracts}

Effects of Mentoring on Career Development and Employee Job Satisfaction

Jamie Blankenship, MLS (ASCP) ${ }^{\mathrm{CM}}$, University of North Carolina Hospital, Chapel Hill, NC

Career development and exploration is an important part of job satisfaction. A mentoring program in the workplace allows for customized career development and interactive education tailored to individual need. A small survey-based study was conducted to evaluate the effectiveness of such a program established in August 2010 in the Core Laboratory, McLendon Clinical Laboratories, UNC Hospital. Participants included 11 technologists in the program from August 2010 to
January 2011. Mentees were matched with a mentor based on interests expressed in their application. Mentees specified goals to address in weekly, hour-long sessions over six months. Two tiers were created to accommodate a wide range of interests. Mentors were named tier I or II based on position, experience and specialization. First tier mentees included new hires and entry level technologists whose goals were to learn more about daily operations. Second tier mentees were more advanced, experienced technologists seeking to learn job functions required for senior positions such as advanced technical skills, project management, and leadership. Participants were allowed to explore skills otherwise inaccessible in their current scope of duties. Evaluation of survey data showed $91 \%$ agreed the program supports their career development. 73\% agreed they met their specified goals for the program. Therefore, a formal mentoring program is an effective way to engage employees by providing individualized career education and leads to overall job satisfaction.

Comparison of Greiner and BD Evacuated Coagulation Blood Collection Tubes

Kathleen Finnegan, MS, MT(ASCP)SH ${ }^{\mathrm{CM}}$, Stony Brook University, Stony Brook, NY

There are several manufacturers of blood collection tubes. Each manufacturer's tube must be validated to ensure that a bias or additional variability is not introduced. This study was initiated to determine the substantial equivalence of hemostasis assays (PT, INR, APTT, fibrinogen, AT, vWF) using Greiner and BD evacuated sodium citrate blood collection tubes. Seventy one volunteers donated two $2.7 \mathrm{ml}$ volume collection tubes (one each from BD and Greiner) and the hemostasis assays were performed using cap piercing technology. Demographic data (age and sex) and warfarin therapy were collected.

Samples were drawn by routine venipuncture and processed for platelet poor plasma within 4 hours of collection adhering to CLSI H21 A5 Collection guidelines. The PT, INR, APTT, fibrinogen, AT and vWF were performed on a Stago STA'-Compact to compare the two tubes with three assay methodologies (viscosity based detection, colorimetric, turbidometric). Assays were performed in singlicate and the values were compared between Greiner and BD tubes by linear 


\section{CLINICAL PRACTICE}

regression analysis. Linear regression analysis demonstrated a correlation between capped Greiner and BD tubes for all assays.

\begin{tabular}{|l|l|l|l|}
\hline \multirow{2}{*}{$\begin{array}{l}\text { Capped } \\
\text { Tubes }\end{array}$} & \multicolumn{4}{|l|}{ Linear Regression } \\
\cline { 2 - 4 } & Slope & Intercept & $\mathrm{R}^{2}$ \\
\hline PT & 0.988 & 0.19 & .993 \\
\hline INR & 0.984 & 0.02 & .992 \\
\hline PTT & 0.966 & 1.00 & .974 \\
\hline Fibrinogen & 0.939 & 12.59 & .888 \\
\hline AT & 0.879 & 11.65 & .792 \\
\hline vWF & 0.951 & 6.66 & .966 \\
\hline
\end{tabular}

The conclusion is that Greiner and BD evacuated blood collection tubes for coagulation testing are substantially equivalent with cap-piercing technology.

Analysis of Bone Mineral Density Biomarkers and Physical Activity in MS

April L Harkins, PhD, MT(ASCP), Alexander Ng, $\mathrm{PhD}, \mathrm{FACSM}$, Benjamin A. Ingraham, Brice Cleland, Molly Pitluck, Paula Papanek, PhD, PT, Marquette University, Milwaukee, WI; Douglas Woo, MD, MaryEllen Csuka, MD, Medical College of Wisconsin, Milwaukee, WI

Multiple sclerosis (MS) patients have increased risk of reduced bone mineral density (BMD) and fractures. There is evidence that low vitamin $\mathrm{D}(25(\mathrm{OH}) \mathrm{D})$ levels and reduced physical activity (PA) negatively affect $\mathrm{BMD}$ in MS. Elevated pro-inflammatory cytokines [i.e. interleukin-6 (IL-6)] and increased cortisol levels have previously been shown to reduce BMD in persons without MS. This study sought to determine associations of vitamin D, PA, endogenous cortisol, cytokines and BMD in MS patients. We tested $21 \mathrm{MS}$ patients and 20 control subjects. The lumbar spine (L2L4) and femoral neck BMD was measured with dual Xray absorptiometry (DXA) and physical activity was measured with accelerometers. Levels of vitamin D, cortisol, and cytokines [IL-6, soluble tumor necrosis factor II (sTNFRII), and interleukin-10 (IL-10)] were measured by RIA or EIA. Analysis was by unpaired ttests and Pearson correlations. The results showed that MS subjects compared to controls had differences with PA ( $\mathrm{p}=0.014)$, IL-6 $(\mathrm{p}=0.01)$ and sTNFRII ( $\mathrm{p}=$ 0.001). No difference was noted in vitamin $D$, cortisol or BMD. Within the MS group alone, PA was also correlated to femoral BMD $(\mathrm{r}=0.56, \mathrm{p}=0.01)$. IL-6 156 VOL 24, NO 3 SUMMER 2011 CLINICAL LABORATORY SCIENCE was correlated to markers of PA within the MS group ( $\mathrm{r}$ $=-0.40, p=0.05)$. We conclude that in MS patients replete with Vitamin D, PA is a contributor to BMD not cortisol, sTNFRII, nor IL-10. IL-6 levels may be a factor in the total physical activity of MS patients.

\section{Evolution of Clinical Practicum Into a University Capstone Course}

Linda H. Jeff, MA, MT(ASCP), Janelle M. Chiasera, $\mathrm{PhD}$, MT(ASCP), The University of Alabama at Birmingham, Birmingham, AL

Inclusion of a quality enhancement plan (QEP) is central to the principles of University accreditation and has become a topic of much discussion. Core requirements specify that Universities develop a carefully designed and comprehensive plan to improve student learning. The University of Alabama at Birmingham (UAB) developed a QEP plan that enhances the undergraduate curriculum by focusing on writing, quantitative literacy, ethics and civic responsibility. The QEP also requires each curriculum to cumulate with a capstone course for students to synthesize and apply what they have learned to real life applications. The Medical Technology clinical practicum was developed into a capstone course by integrating QEP competencies with discipline-specific outcomes. The course includes directed clinical practice in all major disciplines with focused activities to reinforce, integrate and apply knowledge obtained throughout the curriculum. Students organize, build on, and reflect on previous assignments/experiences through the development of a portfolio to demonstrate application of QEP competencies. For example, in one quantitative literacy assignment, students are provided a data set and use a Levey-Jennings chart previously prepared in clinical chemistry laboratory to perform necessary statistics to assess the quality of an analyte over a 30 day period. Attainment of course competencies is evaluated through multiple means including technical and affective evaluation forms and a rubric for portfolio review. Integrating QEP and disciple-specific competencies in a clinical practicum will cultivate in students advanced level skills and depth of knowledge required to be successful leaders in our profession. 


\section{CLINICAL PRACTICE}

Effects of Elevated Fibrinogen, Factor VIII, and von Willebrand factor on the INNOVANCE ${ }^{\circledast}$ PFA P2Y cartridge

David L. McGlasson, 59th Clinical Research Division, Wilford Hall Medical Center, Lackland AFB, TX; Anand D. Shah, Cardiology Division, Wilford Hall Medical Center, Lackland AFB, TX; George A. Fritsma, The Fritsma Factor: Your Interactive Hemostasis Resource

Elevated fibrinogen (Fg), factor VIII (FVIII:C), von Willebrand antigen (VWF:Ag) and von Willebrand factor activity (VWF)have been implicated in thromboembolic events and also in potentially shortening closure times (CTs) of PFA- $100^{\circ}$ (platelet functional analyzer) test cartridges collagen/ADP (Col/ADP) and collagen/epinephrine (Col/EPI). We sought to determine if short P2Y (ADP receptor) CTs (used to detect platelet inhibition induced by clopidogrel [Plavix $\left.{ }^{\oplus}\right]$ ) also correlate with elevated levels of these factors. Blood was collected in $3.2 \%$ citrate from 101 pre-angiography subjects who had received 300-600 mg of clopidogrel within 6-24 hours or 75 mg daily for at least 7 days. Patients were tested with Col/ADP, Col/EPI and P2Y using the PFA- $100^{\circ}$. Patient citrated platelet-poor plasma was tested for $\mathrm{Fg}$, FVIII:C, VWF:Ag and VWF. Mean plasma levels were: Fg 381.0 mg/dl; FVIII:C, 169\%; VWF:Ag, 161\%, VWF, 210\%. Logistic regression yielded the following results:

\begin{tabular}{|l|l|l|l|}
\hline Parameter & \multicolumn{4}{|l|}{ p value } \\
\hline & Col/EPI & Col/ADP & P2Y \\
\hline Fg & 0.61 & 0.63 & 0.68 \\
\hline FVIII:C & 0.68 & 0.88 & 0.50 \\
\hline VWF:Ag & 0.87 & 0.57 & $\mathbf{0 . 0 7}$ \\
\hline VWF & 0.70 & 0.12 & $\mathbf{0 . 0 1}$ \\
\hline
\end{tabular}

Neither Col/EPI nor Col/ADP CTs correlated with elevated factor levels. In contrast, P2Y CTs showed a trend and significant inverse correlation, respectively, with VWF:Ag and VWF. Elevated VWF appears to correlate with shorter P2Y CTs despite clopidogrel therapy. This allows speculation that in addition to elevated VWF, short P2Y CTs may signal risk of thrombotic events in this population. The INNOVANCE ${ }^{\oplus} \mathrm{P} 2 \mathrm{Y}$ cartridge is currently awaiting FDA clearance in the USA.
Microgranular Variant Acute Promyelocytic Leukemia with PML/RARA Gene Rearrangement Nicholas M. Moore, MS, MLS(ASCP) ${ }^{\mathrm{CM}}$, Kindred Healthcare, Inc., Chicago, IL

A 28-year-old male with no significant history presented with five-day history of progressive blurred vision in his right eye. He denied any history of headaches or lightheadedness, but reported bilateral ear pain, sore throat and sinus congestion. He had a decreased appetite, but no weight loss. He was 20/200 upon visual acuity examination, and his retinal exam revealed bilateral macular edema, diffuse retinal hemorrhages and cotton wool spots. He was admitted to the general medical floor. CBC revealed anemia and thrombocytopenia ( $\mathrm{Hb} 5.1 \mathrm{~g} / \mathrm{dL}$, Platelets $9 \times 10^{\wedge} 3 / \mathrm{uL}$ ). The patient was transferred to the MICU for anemia where a central line was inserted and he received two units of packed cells and two of platelets. The peripheral blood smear revealed neutropenia with increased circulating promyelocytes with numerous granules. Flow cytometry revealed immature neutrophils with decreased side scatter based on the CD45/side scatter plot. They expressed CD13 and CD33, but not HLA-DR or CD34. There was partial dim expression of CD117 and CD4, and no CD56 expression. Cytogenetic studies revealed the presence of the RARA gene rearrangement associated with the $(15 ; 17)(\mathrm{q} 22 ; \mathrm{q} 21)$ translocation. Bone Marrow aspirate revealed a hypercellular marrow with an $\mathrm{M}$ : $\mathrm{E}$ ratio of 1.4:1 with increased promyelocytes and no complete maturation of the myeloid line. The patient was started on induction chemotherapy with ATRA along with Decadron and Indarubicin. The patient was discharged home two weeks after his hemoglobin and platelets normalized. Follow-up tests revealed normocellular marrow with proportional trilineage maturation and reveal no signs of residual APL blasts.

Performance Evaluation of the Sysmex XE-2100 Based on Q-Flags from Seven Hematologic Parameters Julie Nelson, MHS, Barbara Kraj, MS, MLS(ASCP) ${ }^{\mathrm{CM}}$, Georgia Health Sciences University, Augusta, GA; Mike Suter, MT(ASCP)SH, Daniel P. Kerrigan, MD, Peacehealth Laboratories, Springfield, OR 


\section{CLINICAL PRACTICE}

Sysmex XE-2100 hematology analyzer recognizes abnormal blood cells and places a warning Q-Flag on the specimen based on trigger points expressed on an arbitrary scale of 0-300. Blasts, left shift, and NRBC have a default trigger setting of 100. Atypical lymphocytes, abnormal lymphocytes/blasts and platelet clumps have a trigger of 125 . Presently, technologists review a significant number of false positive Q-Flags as a result of factory default settings. The purpose of the study was to evaluate the flagging efficiency of seven differential parameters by comparing current instrument's flagging results with manual differentials performed according to Clinical Laboratory Standards Institute H20-A2 protocol. Nonrandomized specimens as ordered by a physician were processed within 24 hours. Of 806 specimens, 703 were flagged using factory-set cut-offs. For any specimen triggering a flag a glass slide was prepared. Efficiency and optimal cut-offs for each parameter were determined using receiveroperating characteristic (ROC) curves. Blast efficiency was $89.7 \%$ and NRBC efficiency was $78.6 \%$ at the default setting; left shift results revealed $83.3 \%$ efficiency at a trigger point of 80 . Atypical lymphocytes showed $79 \%$ efficiency at trigger point of 270 , abnormal lymphocytes/blasts showed peak efficiency of $82.6 \%$ at 290 , and platelet clumps demonstrated $83.3 \%$ efficiency at 300 . Linear regression analysis was used to obtain correlation on percent immature granulocytes. The correlation coefficient was $r=0.5169$. Immature granulocytes, left shift, atypical lymphocytes, abnormal lymphocytes/blasts, and platelet clump flags were candidates for trigger point adjustments while blasts and NRBC flags demonstrated good predictive values at default settings.

Comparison of Humoral Immune Responses of Vaccinia Virus using ELISA and a High Throughput Detection Method

EN McDowell*, NR Patel*, MS, MS Keckler, PhD, IK Damon, PhD, KL Karem,PhD, Centers for Disease Control and Prevention (CDC), Atlanta, GA; *Authors Contributed Equally

Smallpox vaccination induces measurable immunity in both cellular and humoral pathways. One aspect of detecting efficacy of vaccination is to determine the presence of humoral immune responses. Two serologic and diagnostic methods of detecting humoral immunity are ELISA and virus neutralization assay. For this study, standard diagnostic ELISA was used to detect virus specific IgG antibody responses. Virus neutralization was detected by viral fluorescent infection neutralization utilizing a vaccinia vector expressing a green fluorescent protein (GFP) and the arrayscan system, which is an automated fluorescent microscope, coupled with imageprocessing and image analysis that overlays images in order to quantify infectivity. Testing of sera from naïve and primary smallpox vaccinees was performed. Vaccinia immunoglobulin (VIG) was used as a standard positive control. The data demonstrates a correlation of high neutralization titers from VIG relates to a positive IgG antibody response in ELISA. Negative sera display weak neutralization as well as a lack of immunological response by ELISA. Primary vaccinee samples display neutralization titers and IgG ELISA antibody response in a correlative manner. A correlative relationship is also seen in animal models of Orthopoxvirus infection using these two techniques with slight modifications. Combined use of these techniques provides detailed characterization of induced humoral immunity to Orthopoxviruses infection.

\section{The Confirmation of a Novel Approach to Managing} Hemolyzed Specimens

Lester Pretlow, PhD, Shamala Johnson, MHS, CLS, Barbara Russell, EdD, Bridget Evans, MS, Georgia Health Sciences University, Augusta, GA

The Chemistry section of the Medical College of Georgia Hospital, Inc. has instituted unique technologists' duties for managing suboptimal specimens. One technologist, called the driver is responsible for verification, calling criticals, and calling suboptimal specimens to the floors. The driver is assisted by the specimen manager who is responsible for pulling the specimen $\log 3$ to 4 times a shift. The purpose of this study was to determine whether the lab's new approach to managing suboptimal specimens had a significant impact on the turn-around time (TAT) and cost of processing hemolyzed specimens in the laboratory. The investigators queried the laboratory information system for hemolyzed and non-hemolyzed specimens submitted to the chemistry section. The investigators calculated statistical differences for test 


\section{CLINICAL PRACTICE}

TATs and technical costs between four categories of specimens (STAT hemolyzed, STAT nonhemolyzed, routine hemolyzed, and routine nonhemolyzed). Analysis of Variance on the mean TATs and costs for all the groups showed that there was a statistically significant difference in TATs and costs of processing of non-hemolyzed routine specimens versus other categories. T-tests performed between individual specimen categories revealed a statistically significant difference between TATs and cost of STAT hemolyzed specimens versus routine non hemolyzed specimens. However, all hemolyzed specimens were still processed faster than routine specimens and within the section's established goal TAT. The investigators concluded that this ability to process hemolyzed specimens as efficiently as all other categories of specimens was a direct consequence of their new approach to managing suboptimal specimens.

Bacterial Uropathogens and Their Antibiotic Resistance Profile from a Hospital in Central Alabama Li Qian, MD, MT(ASCP), J. Kyle Taylor, MEd, MLS(ASCP) ${ }^{\mathrm{CM}}$, Kathy Jones, MS, MLS(ASCP) ${ }^{\mathrm{CM}}$, Auburn University Montgomery, Montgomery, AL; Tracy Camara, MBA, MT(ASCP)SM, Baptist Medical Center South, Montgomery, AL

Urinary tract infection remains a common problem in inpatient care. It is highly challenging to provide effective initial therapy without sensitivity data. The purpose of this study was to survey the uropathogens and their sensitivity profile at a hospital in Central Alabama to guide experiential antibiotic selection. This was the first reported study on bacterial uropathogens and their antibiotic resistance profile at this Central Alabama hospital. The survey period was between July 2009 and June 2010, a total of 473 urine cultures were reviewed and susceptibility testing was determined using the Clinical and Laboratory Standard Institute(CLSI) microdilution method. The results indicated that Escherichia coli (45.5\%) was the most common organism, followed by Klebsiella pneumoniae (18.2\%), Pseudomonas aeroginosa (10.1\%), Proteus mirabilis (7.8\%), Enterobactor cloacae (4.2\%), Methicillin-Resistant Staphyloccus aureus (3.0\%), Klesiella oxytoca and Citrobacter freundii (1.5\%), Morganella morganii (1.3\%), and the other species
(7.0\%). For the 215 E.coli isolates, imipenem and cephalosporins (except for Cefazolin) had the highest sensitivity (99-100\%, $\mathrm{P}<0.05)$. In contrast, Ampicillin had the highest resistance $(57 \%, \mathrm{P}<0.05)$ as compared to other antibiotics (about 30\%) including ampicillin/sulbactam, ciprofloxacin, levofloxacin, tetracycline, and trimethoprim/sulfamethoxazole. The major finding of this study was that ciprofloxacin, levofloxacin and trimethoprim/sulfamethoxazole had comparable sensitivity patterns for E.coli, K.pneumoniae, P.mirabilis, and E. cloacae, the most common uropathogens at this Central Alabama hospital. Additionally, this study found that $E$. coli had a resistant rate of $31 \%$ to ciprofloxacin and levofloxacin compared to Lee et al. (28.4\%) and Rattanaumpawan et al. $(15.8 \%)$, indicating the continuing evolution of resistance.

Determining the Need to Perform a Manual WBC Differential Count

Barbara Russell, EdD, MT(ASCP)SH, Leanne Kutlik, MHS, MLS(ASCP) ${ }^{\mathrm{CM}}$, Gregory G. Passmore, PhD, CNMT, Lester Pretlow, PhD, C(ASCP) ${ }^{\mathrm{CM}}$, Georgia Health Sciences University, Augusta, GA

Monitoring the white blood cell (WBC) count and absolute neutrophil count (ANC) of oncology patients receiving chemotherapy is essential for ongoing care. Finding a way to provide physicians with WBC counts and ANCs with less turnaround time (TAT) could have a positive impact on patient care. The purpose of this study was to correlate change in WBC to change in ANC for a cross-section of patients undergoing chemotherapy. Data was collected from a convenience population of patients meeting the following criteria: cancer diagnosis, 18 years or older, hospitalized for at least 3 days, and $\mathrm{CBC}$ with differentials performed daily for the entire admission. The WBC, ANC, percentage of segmented neutrophils (segs) and band neutrophils, method of differential count and time/date of each test were recorded. The investigators correlated the data by linear regression to obtain the significance of changes in WBC as related to ANC. The Pearson's Correlation for the change in WBC as related to ANC was 0.9312, the $r^{2}$ was 0.8670 and the significance level was $<0.001$. The investigators calculated regression statistics based on categorizing blood counts according to the elapsed 


\section{CLINICAL PRACTICE}

time to the next test, initial percentage of segs, and amount of change in the WBC. All results showed that there was a statistically significant relationship between changes in the WBC to changes in the ANC for patients undergoing chemotherapy. Therefore, in theory, the change in WBC could be used to predict the ANC which could get test results to the physician faster.

The Use of Immunodiagnostics for the Detection of Invasive Fungal Infections in Immunocompromised Patients: A Case Study

Frank J. Scarano, PhD, Hassan Qadir, MLS(ASCP), University of Massachusetts Dartmouth, North Dartmouth, MA

Patients with hematologic malignancies, and other patients who are immunocompromised for any reason, are at increased risk for invasive fungal infections (IFIs). A major degree of morbidity and mortality is seen in these patients due to these opportunistic IFIs and associated immunosuppressive therapy. Standard diagnosis of fungal infection through mycology culture techniques can be labor intensive and may take several days under the best conditions and up to several weeks in some cases. The use of immunodiagnostics to detect fungal antigens $((1 \rightarrow 3)-\beta$-D-glucan, galactomannan, etc.) in patient serum can diagnose IFIs rapidly (in one day) and up to ten days BEFORE clinical signs and symptoms appear. Early detection of IFIs and intervention with appropriate antifungal therapy significantly decreases the morbidity and mortality in these patients. We present the case of a 51 year old female patient with acute myeloid leukemia, mycobacterial infection and streptococcal septicemia whose IFI was diagnosed and treated early enough to save her life. It is likely that if immunodiagnostics were not used to diagnose her IFI that antifungal therapy would not have been administered early enough and she would have succumbed to the IFI. A review of immunodiagnostic techniques for the detection of IFIs will also be presented.

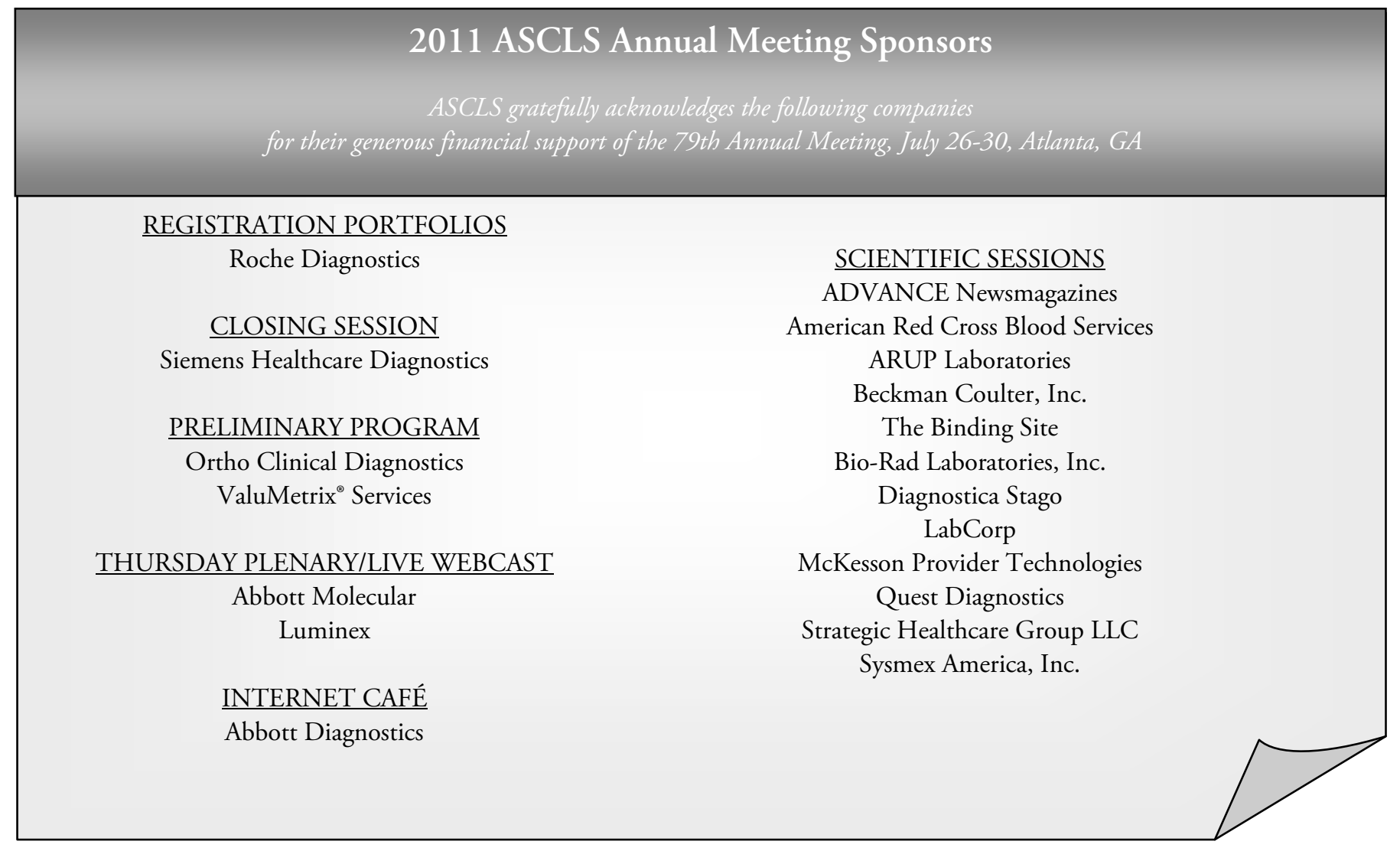

